\title{
В.И. МАРКИН
}

\section{Логика суждений существования и силлогистика*}

\author{
Владимир Ильич Маркин \\ МГУ им. М.В. Ломоносова. \\ Российская Федерация, 119991, г. Москва, Ломоносовский пр-т, д. 27, корп. 4. \\ E-mail: markin@philos.msu.ru
}

Аннотация: В статье строится формальная система, предназначенная для логического анализа суждений существования. В ее языке содержится неопределенно-местная константа существования, а простые формулы образуются сочленением этой константы с произвольной конечной последовательностью общих терминов - положительных (простых) и отрицательных. Сложные формулы образуются с помощью пропозициональных связок. Для данного языка формулируется естественная семантика. Значением (объемом) простого общего термина в некоторой модели является подмножество предметной области данной модели, а объемом отрицательного термина - дополнение к объему соответствующего положительного термина. Простая формула значима в модели, если и только если пересечение объемов общих терминов, из которых она образована, непусто. Предлагается исчисление, аксиоматизирующее класс общезначимых формул. Данное исчисление строится на основе классического исчисления высказываний. В статье обращается внимание на то обстоятельство, что атрибутивные суждения могут быть определены с использованием суждений существования. В связи с этим возникает вопрос о метатеоретических отношениях между построенной логикой и различными силлогистическими теориями. Демонстрируется рекурсивная эквивалентность логики суждений существования и силлогистики с неопределенно-местной константой, которая является обобщением силлогистической константы $a$. С этой целью задается перевод из логики суждений существования в указанную силлогистику и доказывается, что он погружает первую систему во вторую. Задается и обратный перевод, и доказывается, что он также является погружающей операцией. В статье также доказываются метатеоремы о семантической непротиворечивости и полноте исчисления суждений существования.

Ключевые слова: суждения существования, атрибутивные суждения, силлогистика, логическое исчисление, семантика, погружающая операция

Для цитирования: Маркин В.И. Логика суждений существования и силлогистика // Логические исследования / Logical Investigations. 2021. Т. 27. № 2. С. 31-47. DOI: 10.21146/2074-1472-2021-27-2-31-47

* Исследование выполнено в рамках научно-образовательной школы Московского государственного университета имени М.В. Ломоносова «Мозг, когнитивные системы и искусственный интеллект».

Данная статья представляет собой существенно расширенную версию доклада Маркин, 2021, сделанного на Двенадцатых Смирновских чтениях по логике (Москва, 2426 июня 2021 г.).

(C) Маркин В.И. 


\section{1. Введение}

В традиционной логике, наряду с атрибутивными и реляционными, выделялись простые суждения еще одного типа - так называемые суждения существования. Если в атрибутивных суждениях логическим сказуемым (предикатом) является знак свойства (атрибута) индивидов, а в реляционных - знак отношения между индивидами, то в суждениях существования в этой роли выступает термин «существует». Причем данный термин рассматривается не как квантор, а как знак особой онтологической характеристики индивидов.

Суждения существования - так же как простые суждения других видов - делят (по качеству) на утвердительные и отрицательные: в утвердительных фиксируется существование объекта (объектов), а в отрицательных - его (их) несуществование. Например, суждение «Черные лебеди существуют» утвердительное, а суждение «Флогистон не существует» отрицательное.

Суждения существования можно, в принципе, разделить и по количеству: на единичные и множественные. Основанием этого деления выступает тип их логического подлежащего (субъекта). Субъектом единичного суждения существования является сингулярный термин («флогистон»). В качестве субъектов множественных суждений существования могут выступать: простые (положительные) общие термины («млекопитающие»), отрицательные общие термины («беспозвоночные»), а также последовательности нескольких общих терминов («фруктоядные рукокрылые млекопитающие») .

В истории логики встречается точка зрения, согласно которой статус суждений существования более фундаментален по сравнению с атрибутивными (категорическими) суждениями в том отношении, что вторые можно - не меняя их смысла - редуцировать к первым.

Так, Франц Брентано считал, что суждение формы «Все $S$ есть $P$ эквивалентно суждению существования «Sне- $P$ не существуют», суждение формы «Некоторые $S$ есть $P$ - суждению «SP существуют», суждение формы «Ни один $S$ не есть $P »-$ суждению «SP не существуют», а суждение формы «Некоторые $S$ не есть $P »-$ суждению «Sне- $P$ существуют» (см. Brandl, 2018).

Сходной позиции придерживался и Льюис Кэрролл Carroll, 1896. При анализе силлогизмов он также интерпретировал категорические суждения как суждения существования. Интерпретация частноутвердительных и общеотрицательных суждений у него аналогична брентановской: суждение «Некоторые купцы являются скупцами» выражает ту же мысль, что и суждение «Купцы-скупцы существуют», а суждение «Ни одна русалка не 
является модисткой» - ту же мысль, что и суждение «Русалок-модисток не существует». Общеутвердительное суждение формы «Все $S$ есть $P$ », согласно Кэрроллу, эквивалентно конъюнкции двух суждений существования: «SP существуют» и «Sне- $P$ не существуют». Например, суждение «Все банкиры богаты» выражает ту же мысль, что и «Богатые банкиры существуют, а небогатых банкиров не существует». Частноотрицательные суждения «Некоторые $S$ не есть $P$ » Кэрроллом не рассматривались, он считал их равнозначными суждениям формы «Некоторые $S$ есть не- $P$ ».

Наиболее ярко связь категорических суждений с суждениями существования проявляется у Кэрролла в предложенном им для проверки силлогизмов диаграммном методе. Информация суждения «Некоторые $S$ есть $P$ » выражается постановкой красной фишки в сектор $S P$, что означает существование элемента в пересечении объемов $S$ и $P$. Информация суждения «Ни один $S$ не есть $P$ » выражается постановкой черной фишки в сектор $S P$, что указывает на пустоту пересечения объемов $S$ и $P$. Информация суждения «Все $S$ есть $P$ » передается постановкой красной фишки в сектор $S P$ и черной фишки в сектор $S$ не- $P$, таким образом, пересечение объемов $S$ и $P$ объявляется непустым, а пересечение объемов $S$ и не- $P$ пустым.

На трехбуквенных диаграммах Кэрролла атрибутивные суждения сводятся, по существу, к булевым комбинациям суждений существования, субъектами которых являются последовательности трех общих терминов. Так, информацию суждения «Все $S$ есть $M »$ он выражает постановкой красной фишки на границу секторов $S M P$ и $S M$ не- $P$ и помещением черных фишек в сектора $S$ не- $M P$ и $S$ не- $M$ не- $P$. При этом «Все $S$ есть $M$ » оказывается эквивалентным сложному суждению «SMP или $S M$ не- $P$ существуют, а $S$ не- $M P$ и $S$ не- $M$ не- $P$ не существуют». Первая часть этого сложного суждения, согласно Кэрроллу, равносильна суждению «SM существуют», а вторая часть - суждению «Sне- $M$ не существуют».

В данной работе мы сформулируем логическую систему, в языке которой фиксируются логические формы множественных суждений существования, субъектами которых являются общие термины или их последовательности. Для данного языка мы предложим естественную теоретикомножественную семантику и построим исчисление, аксиоматизирующее класс общезначимых в этой семантике формул.

Далее будет доказана рекурсивная эквивалентность логики суждений существования и построенной в [Маркин, 2004 силлогистики с неопределенно-местной константой, которая является обобщением силлогистической константы $a$. 
В заключении будет рассмотрен вопрос о соотношении логики суждений существования и дедуктивных теорий, сформулированных в стандартном силлогистическом языке.

\section{2. Логика суждений существования: язык, семантика, аксиоматическое исчисление}

Логику суждений существования будем строить на базе классической логики высказываний, как это принято при современных формализациях силлогистических теорий.

В алфавите языка содержатся: бесконечный список простых общих терминов (будем использовать для них метапеременные $S, P, M, S_{1}, \ldots$ ), символ терминного отрицания ('), неопределенно-местная константа существования $(\Upsilon)$, пропозициональные связки и скобки.

Общими терминами являются: (1) произвольный простой общий термин, (2) выражение вида $S^{\prime}$, где $S$ - простой общий термин. При этом $S$ будем называть положительным, а $S^{\prime}$ - отрицательным термином. В качестве метапеременных по любым общим терминам (как положительным, так и отрицательным) будем использовать символы $X, Z, X_{1}, \ldots$

Атомарными формулами языка нашей системы являются выражения вида $\Upsilon X_{1} X_{2} \ldots X_{k}(k \geq 1)$, где $X_{1}, X_{2}, \ldots X_{k}$ - общие термины. Формула $\Upsilon X_{1} X_{2} \ldots X_{k}$ фиксирует логическую форму суждения существования « $X_{1} X_{2} \ldots X_{k}$ существуют». Сложные формуль образуются из других формул с помощью пропозициональных связок.

Для данного языка зададим точную семантику. Моделью называется пара $\langle\mathbf{D}, \varphi\rangle$, где $\mathbf{D} \neq \varnothing$, а $\varphi(S) \subseteq \mathbf{D}$ для любого простого общего термина $S$. Определим функцию $\psi$, сопоставляющую значение каждому общему термину (включая отрицательные) в модели $\langle\mathbf{D}, \varphi\rangle: \psi(S)=\varphi(S), \psi\left(S^{\prime}\right)=$ $\mathbf{D} \backslash \varphi(S) . \psi(X)$ представляет собой подмножество предметной области $\mathbf{D}$ и называется объемом общего термина $X$.

Введем далее понятие $\mathcal{V}$-значимости формулы нашего языка в модели $\langle\mathbf{D}, \varphi\rangle$. Условия значимости атомарных формул определяются так:

$$
\mathcal{V}\left(\Upsilon X_{1} X_{2} \ldots X_{k}, \mathbf{D}, \varphi\right) \text {, е.т.е. } \psi\left(X_{1}\right) \cap \psi\left(X_{2}\right) \cap \ldots \cap \psi\left(X_{k}\right) \neq \varnothing .
$$

Данное семантическое определение соответствует естественной трактовке суждений существования: суждение « $X_{1} X_{2} \ldots X_{k}$ существуют» истинно тогда и только тогда, когда найдется объект, принадлежащий объемам всех общих терминов $X_{1}, X_{2}, \ldots X_{k}$.

Условия значимости формул, образованных с помощью пропозициональных связок, стандартные. 
Формула $A$ называется $\mathcal{V}$-общезначимой, е.т.е. $\mathcal{V}(A, \mathbf{D}, \varphi)$ в каждой модели $\langle\mathbf{D}, \varphi\rangle$.

Ниже будет доказано, что класс $\mathcal{V}$-общезначимых формул аксиоматизирует исчисление $\mathbf{C} \boldsymbol{\Upsilon}$. Оно содержит схемы аксиом классического исчисления высказываний и шесть дополнительных схем аксиом:

$\Upsilon 1 . \Upsilon u v \supset\left(\Upsilon u M^{\prime} \vee \Upsilon M v\right)$

$\Upsilon 2 . \neg \Upsilon S S^{\prime}$,

ฯ3. $u X Z v \supset \Upsilon u Z X v$,

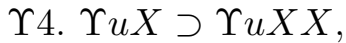

$\Upsilon 5 . \Upsilon w X \supset \Upsilon w$

$\Upsilon 6 . \Upsilon S \vee \Upsilon S^{\prime}$,

где $u$ и $v$ - конечные и, возможно, пустые последовательности общих терминов (в 1 по крайней мере одна из них непуста), $w$ - непустая конечная последовательность общих терминов, $S$ и $M$ - простые общие термины, $X$ и $Z$ - произвольные общие термины.

Единственным правилом вывода в $\mathbf{C} \Upsilon$ является modus ponens. Понятия доказательства и теоремы стандартные.

\section{3. Силлогистика с неопределенно-местной константой}

Осуществим сравнение логики суждений существования $\mathbf{C} \mathbf{\Upsilon}$ с силлогистикой СФ@, сформулированной в Маркин, 2004. В последней решается проблема силлогистического представления всех возможных отношений между объемами произвольного конечного числа простых общих терминов в некотором универсуме без введения в язык терминообразующих операторов, т.е. в рамках позитивной силлогистики, где не рассматриваются отрицательные и другие сложные термины.

Язык СФ@ содержит неопределенно-местную силлогистическую константу @, а также бесконечный список простых общих терминов, пропозициональные связки и скобки. Атомарные формулы этого силлогистического языка имеют вид $S_{1} S_{2} \ldots S_{n} @ P_{1} P_{2} \ldots P_{m}$, где $S_{1}, S_{2}, \ldots, S_{n}, P_{1}, P_{2}, \ldots, P_{m}$ простые общие термины и $n+m \geq 1$. Сложные формулы образуются с помощью пропозициональных связок.

В семантике данного языка используются модели того же типа, что и в сформулированной ранее логике суждений существования. Вводится понятие $\mathcal{W}$-значимости формул языка $\mathbf{C \Phi} @$ в модели $\langle\mathbf{D}, \varphi\rangle$. Условия $\mathcal{W}$-значимости атомарных формул определяются следующим образом:

$$
\begin{gathered}
\mathcal{W}\left(S_{1} S_{2} \ldots S_{n} @ P_{1} P_{2} \ldots P_{m}, \mathbf{D}, \varphi\right), \text { e.r.e. } \\
\varphi\left(S_{1}\right) \cap \varphi\left(S_{2}\right) \cap \ldots \cap \varphi\left(S_{n}\right) \cap\left(\mathbf{D} \backslash \varphi\left(P_{1}\right)\right) \cap\left(\mathbf{D} \backslash \varphi\left(P_{2}\right)\right) \cap \ldots \cap\left(\mathbf{D} \backslash \varphi\left(P_{m}\right)\right)=\varnothing .
\end{gathered}
$$


Таким образом, формула вида $S_{1} S_{2} \ldots S_{n} @ P_{1} P_{2} \ldots P_{m}$ значима в том и только в том случае, когда пересечение объемов $S_{1}, S_{2}, \ldots, S_{n}$ и антиобъемов $P_{1}$, $P_{2}, \ldots, P_{m}$ пусто.

Условия $\mathcal{W}$-значимости сложных формул обычные.

Форма общеутвердительного суждения $(S a P)$ при его стандартной трактовке как суждения о включенности объема субъекта в объем предиката выражается в этой силлогистике формулой $S @ P$. Поэтому символ @ можно истолковать как своего рода обобщение обычной силлогистической константы $a$.

Форма общеотрицательного суждения $(\mathrm{SeP})$, стандартно понимаемого как суждение об объемной несовместимости субъекта и предиката, выражается здесь формулой $S P @$. Формуле $S i P$ обычного языка силлогистики соответствует $\neg S P @$, а формуле $S o P-\neg S @ P$.

Формула $B$ силлогистического языка (с константой @) $\mathcal{W}$-общезначима, е.т.е. $\mathcal{W}(B, D, \varphi)$ в каждой модели $\langle\mathbf{D}, \varphi\rangle$.

Класс $\mathcal{W}$-общезначимых формул аксиоматизирует исчисление $\mathbf{C \Phi} @$ При формальной записи его постулатов $s, p, q, r$ обозначают последовательности (возможно, пустые) общих терминов. Помимо схем аксиом классического исчисления высказываний, в $\mathbf{C \Phi} @$ постулируются следующие силлогистические схемы аксиом:

@1. $(M q @ r \wedge s @ p M) \supset s q @ p r$,

@2. $S @ S$,

@3. $s S P p @ q \supset s P S p @ q$,

@4. $s @ p S P q \supset s @ p P S q$,

@5. $S S s @ p \supset S s @ p$,

@6. $s @ p P P \supset s @ p P$,

@7. $s @ p \supset S s @ p$,

@8. $s @ p \supset s @ p P$,

@9. $\neg(S @ \wedge @ S)$.

В @1 по крайней мере одна из последовательностей терминов $-s, q, p$ или $r$ - должна быть непустой. В @7 и @8 непуста хотя бы одна из последовательностей $-s$ или $p$.

Единственное правило вывода в $\mathbf{C \Phi} @$ - modus ponens.

В Маркин, 2004] доказано, что множество теорем исчисления СФ@ и множество $\mathcal{W}$-общезначимых формул совпадают. 


\section{4. Рекурсивная эквивалентность С $\Upsilon$ и $\mathrm{C \Phi @}$}

Сравнение исчислений $\mathbf{C} \mathbf{\Upsilon}$ и $\mathbf{C \Phi} @$ будем осуществлять в терминах nогружающ, операчий. Нам предстоит доказать, что эти системы погружаются друг в друга, т.е. рекурсивно эквивалентны.

Зададим функцию перевода $\Omega_{1}$, сопоставляющую каждой формуле $A$ языка $\mathbf{C} \boldsymbol{\Upsilon}$ силлогистическую формулу $\Omega_{1}(A)$ языка $\mathbf{C} \boldsymbol{\Phi} @$

Пусть $u$ - произвольная непустая конечная последовательность общих терминов. Пусть $S_{1}, S_{2}, \ldots, S_{n}$ - последовательность, получающаяся из $u$ вычеркиванием всех отрицательных терминов, а $P_{1}^{\prime}, P_{2}^{\prime}, \ldots, P_{m}^{\prime}-$ последовательность, получающаяся из $u$ вычеркиванием всех положительных терминов. Тогда перевод атомарной формулы $\Upsilon$ определяется так:

$$
\Omega_{1}(\Upsilon u)=\neg S_{1} S_{2} \ldots S_{n} @ P_{1} P_{2} \ldots P_{m} .
$$

На сложные формулы функция $\Omega_{1}$ распространяется следующим образом:

$$
\Omega_{1}(\neg C)=\neg \Omega_{1}(C), \quad \Omega_{1}(C \nabla D)=\Omega_{1}(C) \nabla \Omega_{1}(D),
$$

где $\nabla$ - любая бинарная пропозициональная связка.

Зададим также функцию перевода $\Omega_{2}$, сопоставляющую каждой силлогистической формуле $B$ языка $\mathbf{C \Phi} @$ формулу $\Omega_{2}(B)$ языка $\mathbf{C \Upsilon}$ :

$$
\begin{aligned}
& \Omega_{2}\left(S_{1} S_{2} \ldots S_{n} @ P_{1} P_{2} \ldots P_{m}\right)=\neg \Upsilon S_{1} S_{2} \ldots S_{n} P_{1}^{\prime} P_{2}^{\prime} \ldots P_{m}^{\prime}, \\
& \Omega_{2}(\neg C)=\neg \Omega_{2}(C), \quad \Omega_{2}(C \nabla D)=\Omega_{2}(C) \nabla \Omega_{2}(D) .
\end{aligned}
$$

Предварительно обоснуем справедливость ряда метаутверждений.

Теорема 1. Для любой формулы А языка $\mathbf{C \Upsilon}$ верно, что если она доказуема в $\mathbf{C \Upsilon}$, то ее перевод - формула $\Omega_{1}(A)$ - доказуем в $\mathbf{C \Phi} @$.

Доказательство. Рассмотрим произвольную формулу $A$, доказуемую в C؟. Покажем, что $\Omega_{1}$-переводы всех формул, входящих в состав доказательства $A$ в $\mathbf{C} \boldsymbol{\Upsilon}$ (в том числе и последней формулы этой последовательности, т.е. самой $A$ ), доказуемы в $\mathbf{C \Phi} @$. Для этого нужно убедиться, во-первых, в том, что $\Omega_{1}$-переводы всех аксиом системы $\mathbf{C} \boldsymbol{\Upsilon}$ доказуемы в $\mathbf{C \Phi} @$, а во-вторых, что если $\Omega_{1}$-переводы посылок правила modus ponens доказуемы в $\mathbf{C \Phi} @$, то и $\Omega_{1}$-перевод его заключения также доказуем в $\mathbf{C} \boldsymbol{\Phi} @$.

$\Upsilon 1 . \Omega_{1}\left(\Upsilon u v \supset\left(\Upsilon u M^{\prime} \vee \Upsilon M v\right)\right)=\neg s q @ p r \supset(\neg s @ p M \vee \neg M q @ r)$, где $s$ и $q-$ последовательности положительных терминов в составе, соответственно, $u$ и $v$, а $p$ и $r$ получаются удалением терминных отрицаний из последовательностей отрицательных терминов в составе $u$ и $v$. Выводится из @1 (( $M q @ r \wedge s @ p M) \supset s q @ p r)$ по законам классической логики высказываний (ЛВ). 
$\Upsilon 2 . \Omega_{1}\left(\neg \Upsilon S S^{\prime}\right)=\neg \neg S @ S$.

Выводится из $@ 2(S @ S)$ по законам ЛВ.

$\Upsilon 3$. Следует рассмотреть четыре случая в зависимости от того, положительными или отрицательными являются термины $X$ и $Z$ в схеме $\Upsilon u X Z v \supset \Upsilon u Z X v$. Пусть последовательности $s, q, p$ и $r$ определяются так же, как и в пункте $(\Upsilon 1)$.

(1) $X$ и $Z$ - положительные термины (напр., $S$ и $P$ ). Тогда $\Omega_{1}(\Upsilon u X Z v \supset \Upsilon u Z X v)=\neg s S P q @ p r \supset \neg s P S q @ p r$. Формула $\neg s S P q @ p r \supset$ $\neg s P S q @ p r$ выводится из $s P S q @ p r \supset s S P q @ p r$ (варианта @3) по контрапозиции.

(2) $X$ и $Z-$ отрицательные термины (напр., $S^{\prime}$ и $P^{\prime}$ ). Тогда $\Omega_{1}(\Upsilon u X Z v \supset \Upsilon u Z X v)=\neg s q @ p S P r \supset \neg s q @ p P S r$. Формула $\neg s q @ p S P r \supset$ $\neg s q @ p P S r$ выводится из $s q @ p P S r \supset s q @ p S P r$ (варианта @4) по контрапозиции.

(3) $X$ - положительный термин (напр., $S$ ), а $Z$ - отрицательный термин (напр., $\left.P^{\prime}\right)$. Тогда $\Omega_{1}(\Upsilon u X Z v \supset \Upsilon u Z X v)=\neg s S q @ p P r \neg s S q @ p P r$. Это закон тождества ЛВ.

(4) $X$ - отрицательный термин (напр., $S^{\prime}$ ), а $Z$ - положительный термин (напр., $P)$. Тогда $\Omega_{1}(\Upsilon u X Z v \supset \Upsilon u Z X v)=\neg s P q @ p S r \supset \neg s P q @ p S r$. Это закон тождества ЛВ.

$\Upsilon 4$. Пусть $s$ - последовательность положительных терминов в составе $u$, а $p$ получается удалением терминных отрицаний из последовательности отрицательных терминов в составе $u$.

Если $X$ - положительный термин (напр., $S)$, то $\Omega_{1}(\Upsilon u X \supset \Upsilon u X X)=$ $\neg s S @ p \supset \neg s S S @ p$. Формула $\neg s S @ p \supset \neg s S S @ p$ получается из @5 $(S S s @ p \supset$ $S s @ p)$ с использованием законов ЛВ, а также аксиом «перестановки» @3.

Если $X$ - отрицательный термин (напр., $\left.P^{\prime}\right)$, то $\Omega_{1}(\Upsilon u X \supset \Upsilon u X X)=$ $\neg s @ p P \supset \neg s @ p P P$. Формула $\neg s @ p P \supset \neg s @ p P P$ получается из @6 $(s @ p P P \supset s @ p P)$ по контрапозиции.

$\Upsilon 5$. Пусть $s-$ последовательность положительных терминов в составе $w$, а $p$ получается удалением терминных отрицаний из последовательности отрицательных терминов в составе $w$.

Если $X-$ положительный термин (напр., $S)$, то $\Omega_{1}(\Upsilon w X \supset \Upsilon w)=$ $\neg s S @ p \supset \neg s @ p$. Формула $\neg s S @ p \supset \neg s @ p$ получается из @7 $(s @ p \supset S s @ p)$ с использованием законов ЛВ, а также аксиом «перестановки»@3.

Если $X-$ отрицательный термин (напр., $\left.P^{\prime}\right)$, то $\Omega_{1}(\Upsilon w X \supset \Upsilon w)=$ $\neg s @ p P \supset \neg s @ p$. Формула $\neg s @ p P \supset \neg s @ p$ получается из @8 $(s @ p \supset s @ p P)$ по контрапозиции.

$\Upsilon 6 . \Omega_{1}\left(\Upsilon S \vee \Upsilon S^{\prime}\right)=\neg @ S \vee \neg S @$.

Выводится из @9 $(\neg(S @ \wedge @ S))$ по законам ЛВ. 
MP. Пусть $\Omega_{1}$-переводы формул $A \supset B$ и $A$ языка $\mathbf{C} \boldsymbol{\Upsilon}$ доказуемы в $\mathbf{C \Phi} @$. Поскольку по определению $\Omega_{1} \Omega_{1}(A \supset B)=\Omega_{1}(A) \supset \Omega_{1}(B)$, формула $\Omega_{1}(A) \supset \Omega_{1}(B)$ доказуема в этой системе. Но и $\Omega_{1}(A)$ в ней доказуема. Применяя МР к последним двум формулам в $\mathbf{C \Phi} @$, получаем, что $\Omega_{1}(B)$ также доказуема в данном исчислении.

Теорема 2. Для любой формуль $B$ языка $\mathbf{C \Phi @ ~ в е р н о , ~ ч т о ~ е с л и ~ о н а ~ д о к а - ~}$ зуема в $\mathbf{C \Phi} @$, то ее перевод - формула $\Omega_{2}(B)$ - доказуем в $\mathbf{C} \mathbf{\Upsilon}$.

Доказательство. Применяем тот же метод, что и при доказательстве Теоремы 1 .

Пусть $s, q, p$ и $r$ - произвольные последовательности, состоящие из простых (положительных) общих терминов. Символами $\bar{s}, \bar{q}, \bar{p}$ и $\bar{r}$ будем обозначать последовательности, образующиеся из $s, q, p$ и $r$ заменой каждого положительного термина в их составе на соответствующий отрицательный (напр., $S$ на $S^{\prime}$ ).

@1. $\Omega_{2}((M q @ r \wedge s @ p M) \supset s q @ p r)=\left(\neg \Upsilon M q \bar{r} \wedge \neg \Upsilon s \bar{p} M^{\prime}\right) \supset \neg \Upsilon s q \bar{p} \bar{r}$.

Доказываем в $\mathbf{C}$ сначала формулу $\Upsilon s q \bar{p} \bar{r} \supset \Upsilon s \bar{p} q \bar{r}$, используя нужное число раз аксиомы «перестановки» $(\Upsilon 3)$ и законы транзитивности $\supset$. Далее используем вариант аксиомы $\Upsilon 1-$ формулу $\Upsilon s \bar{p} q \bar{r} \supset\left(\Upsilon s \bar{p} M^{\prime} \vee\right.$ $\Upsilon M q \bar{r})$. Из этих двух формул по законам ЛВ выводится искомая формула $\left(\neg \Upsilon M q \bar{r} \wedge \neg \Upsilon s \bar{p} M^{\prime}\right) \supset \neg \Upsilon s q \bar{p} \bar{r}$.

@2. $\Omega_{2}(S @ S)=\neg \Upsilon S S^{\prime}$. Это аксиома $\Upsilon 2$.

@3. $\Omega_{2}(s S P p @ q \supset s P S p @ q)=\neg \Upsilon s S P p \bar{q} \supset \neg \Upsilon s P S p \bar{q}$.

Выводится из $\Upsilon s P S p \bar{q} \supset \Upsilon s S P p \bar{q}$ (варианта $\Upsilon 3$ ) по контрапозиции.

$@ 4 . \Omega_{2}(s @ p S P q \supset s @ p P S q)=\neg \Upsilon s \bar{p} S^{\prime} P^{\prime} \bar{q} \supset \neg \Upsilon s \bar{p} P^{\prime} S^{\prime} \bar{q}$.

Выводится из $\Upsilon s \bar{p} P^{\prime} S^{\prime} \bar{q} \supset \Upsilon s \bar{p} S^{\prime} P^{\prime} \bar{q}$ (варианта $\Upsilon 3$ ) по контрапозиции.

@5. $\Omega_{2}(S S s @ p \supset S s @ p)=\neg \Upsilon S S s \bar{p} \supset \neg \Upsilon S s \bar{p}$.

Выводится из $\Upsilon s \bar{p} S \supset \Upsilon s \bar{p} S S$ (варианта $\Upsilon 4$ ) с помощью аксиом «перестановки» $(\Upsilon 3)$ и законов ЛВ.

@6. $\Omega_{2}(s @ p P P \supset s @ p P)=\neg \Upsilon s \bar{p} P^{\prime} P^{\prime} \supset \neg \Upsilon s \bar{p} P^{\prime}$.

Выводится из $\Upsilon s \bar{p} P^{\prime} \supset \Upsilon s \bar{p} P^{\prime} P^{\prime}$ (варианта $\Upsilon 4$ ) по контрапозиции.

@7. $\Omega_{2}(s @ p \supset S s @ p)=\neg \Upsilon s \bar{p} \supset \neg \Upsilon S s \bar{p}$.

Выводится из $\Upsilon s \bar{p} S \supset \Upsilon s \bar{p}$ (варианта $\Upsilon 5)$ с помощью аксиом «перестановки» $(\Upsilon 3)$ и законов ЛВ.

@8. $\Omega_{2}(s @ p \supset s @ p P)=\neg \Upsilon s \bar{p} \supset \neg \Upsilon s \bar{p} P^{\prime}$.

Выводится из $\Upsilon s \bar{p} P^{\prime} \supset \Upsilon s \bar{p}$ (варианта $\left.\Upsilon 5\right)$ по контрапозиции.

@9. $\Omega_{2}(\neg(S @ \wedge @ S))=\neg\left(\neg \Upsilon S \wedge \neg \Upsilon S^{\prime}\right)$.

Выводится из $\Upsilon 6\left(\Upsilon S \vee \Upsilon S^{\prime}\right)$ по законам ЛВ.

MP. Обосновывается аналогично последнему пункту Теоремы 1. 
Теорема 3. Формула $A \equiv \Omega_{2}\left(\Omega_{1}(A)\right)$ является теоремой $\mathbf{C} \boldsymbol{\Upsilon}$ для любой формулы $А$ языка данного исчисления.

Доказательство. Применяем возвратную индукцию по количеству пропозициональных связок в составе формулы $A$ языка $\mathbf{C}$.

Рассмотрим сначала базисный случай, когда $A$ - атомарная формула, т.е. имеет вид $\Upsilon u$, где $u$ - непустая конечная последовательность общих терминов. Выделим в составе $u$ последовательность $S_{1}, S_{2}, \ldots, S_{n}$ положительных терминов и последовательность $P_{1}^{\prime}, P_{2}^{\prime}, \ldots, P_{m}^{\prime}$ отрицательных терминов (причем эти термины расположим в том же порядке, что и в $u$ ). По определению перевода $\Omega_{1} \Omega_{1}(A)=\neg S_{1} S_{2} \ldots S_{n} @ P_{1} P_{2} \ldots P_{m}$. Тогда по определению перевода $\Omega_{2} \Omega_{2}\left(\Omega_{1}(A)\right)=\neg \neg \Upsilon S_{1} S_{2} \ldots S_{n} P_{1}^{\prime} P_{2}^{\prime} \ldots P_{m}^{\prime}$. С использованием аксиом схемы $\Upsilon 3$ системы $\mathbf{C} \Upsilon$ несложно доказать в ней формулу $\Upsilon u \equiv \Upsilon S_{1} S_{2} \ldots S_{n} P_{1}^{\prime} P_{2}^{\prime} \ldots P_{m}^{\prime}$. Отсюда по законам ЛВ вытекает, что и формула $\Upsilon u \equiv \neg \neg \Upsilon S_{1} S_{2} \ldots S_{n} P_{1}^{\prime} P_{2}^{\prime} \ldots P_{m}^{\prime}$ доказуема в $\mathbf{C} \Upsilon$.

Обоснуем далее индуктивный переход. Допустим, что для любых формул $C$ и $D$, содержащих меньше пропозициональных связок, чем $A$, утверждение теоремы верно, т.е. формулы $C \equiv \Omega_{2}\left(\Omega_{1}(C)\right)$ и $D \equiv \Omega_{2}\left(\Omega_{1}(D)\right)$ доказуемы в $\mathbf{C} \boldsymbol{\Upsilon}$.

Пусть $A=\neg C$. Поскольку $C \equiv \Omega_{2}\left(\Omega_{1}(C)\right)$ доказуема в $\mathbf{C} \mathbf{\Upsilon}$ по индуктивному допущению, постольку (в силу законов ЛВ) формула $\neg C \equiv$ $\neg \Omega_{2}\left(\Omega_{1}(C)\right)$ также доказуема в этой системе. Но по определению переводов $\Omega_{1}$ и $\Omega_{2}$ имеем: $\neg \Omega_{2}\left(\Omega_{1}(C)\right)=\Omega_{2}\left(\neg \Omega_{1}(C)\right)=\Omega_{2}\left(\Omega_{1}(\neg C)\right)$.

Пусть $A=C \wedge D$. Из допущения о доказуемости формул $C \equiv \Omega_{2}\left(\Omega_{1}(C)\right)$ и $D \equiv \Omega_{2}\left(\Omega_{1}(D)\right)$ по законам ЛВ вытекает, что формула $(C \wedge D) \equiv$ $\left.\Omega_{2}\left(\Omega_{1}(C)\right) \wedge \Omega_{2}\left(\Omega_{1}(D)\right)\right)$ доказуема в $\mathbf{C} \boldsymbol{\Upsilon}$. Но по определению переводов $\Omega_{1}$ и $\Omega_{2}$ имеем: $\Omega_{2}\left(\Omega_{1}(C)\right) \wedge \Omega_{2}\left(\Omega_{1}(D)\right)=\Omega_{2}\left(\Omega_{1}(C) \wedge \Omega_{1}(D)\right)=\Omega_{2}\left(\Omega_{1}(C \wedge D)\right)$.

Для сложных формул, образованных с помощью иных бинарных пропозициональных связок, рассуждаем аналогично.

Теорема 4. Формула $B \equiv \Omega_{1}\left(\Omega_{2}(B)\right)$ является теоремой $\mathbf{C \Phi} @$ для любой формулы $B$ языка данного исчисления.

Доказателъство. Применяем возвратную индукцию по количеству пропозициональных связок в составе формулы $B$ языка $\mathbf{C \Phi} @$.

Пусть $B-$ атомарная формула языка $\mathbf{C \Phi} @$, т.е. имеет вид $S_{1} S_{2} \ldots S_{n} @ P_{1} P_{2} \ldots P_{m}$. По определению перевода $\Omega_{2} \Omega_{2}(B)=$ $\neg \Upsilon S_{1} S_{2} \ldots S_{n} P_{1}^{\prime} P_{2}^{\prime} \ldots P_{m}^{\prime}$. Тогда, по определению перевода $\Omega_{1}, \Omega_{1}\left(\Omega_{2}(B)\right)=$ $\neg \neg S_{1} S_{2} \ldots S_{n} @ P_{1} P_{2} \ldots P_{m}$. Формула $B \equiv \Omega_{1}\left(\Omega_{2}(B)\right)$ в этом случае оказывается пропозициональной тавтологией $(B \equiv \neg \neg B)$.

Обоснование индуктивного перехода аналогично соответствующему фрагменту доказательства Теоремы 3. 
Из только что доказанных теорем, согласно известному критерию В.А. Смирнова [Смирнов, 2002, с. 122], непосредственно вытекают два важных результата:

Теорема 5. Перевод $\Omega_{1}$ погружает исчисление $\mathbf{C \Upsilon}$ в $\mathbf{C \Phi @ . ~}$

Теорема 6. Перевод $\Omega_{2}$ погружает исчисление $\mathbf{C \Phi @ ~ в ~} \mathbf{C \Upsilon}$.

Таким образом, доказано, что исчисление суждений существования $\mathbf{C} \mathbf{\Upsilon}$ и исчисление $\mathbf{C \Phi} @$ с неопределенно-местной силлогистической константой рекурсивно эквивалентны.

\section{5. Семантическая непротиворечивость и полнота С $\Upsilon$}

Опираясь на результат о погружаемости $\mathbf{C} \mathbf{\Upsilon}$ в $\mathbf{C \Phi} @$, можно достаточно просто доказать, что исчисление $\mathbf{C} \boldsymbol{\Upsilon}$ является адекватной формализацией логики суждений существования, семантически построенной в Разделе 2.

Сравнивая условия $\mathcal{V}$-значимости произвольной формулы $A$ языка $\mathbf{C} \boldsymbol{\Upsilon}$ в модели $\langle\mathbf{D}, \varphi\rangle$ и условия $\mathcal{W}$-значимости в той же модели ее $\Omega_{1}$-перевода, предварительно обоснуем следующее метаутверждение:

Теорема 7. Для любой формуль $A$ языка $\mathbf{C} \boldsymbol{\Upsilon}$ и каждой модели $\langle\mathbf{D}, \varphi\rangle$ верно, что $\mathcal{V}(A, \mathbf{D}, \varphi)$, е.т.е. $\mathcal{W}\left(\Omega_{1}(A), \mathbf{D}, \varphi\right)$.

Доказателъство. Применяем возвратную индукцию по количеству пропозициональных связок в составе формулы $A$ языка $\mathbf{C}$.

Пусть $A$ - атомарная формула, т.е. формула вида $\Upsilon X_{1} X_{2} \ldots X_{k}$. Она является $\mathcal{V}$-значимой, е.т.е. $\psi\left(X_{1}\right) \cap \psi\left(X_{2}\right) \cap \ldots \cap \psi\left(X_{k}\right) \neq \varnothing$. Выделим в списке общих терминов $X_{1}, X_{2}, \ldots, X_{k}$ положительные (пусть ими будут $S_{1}$, $\left.S_{2}, \ldots, S_{n}\right)$ и отрицательные $\left(P_{1}^{\prime}, P_{2}^{\prime}, \ldots, P_{m}^{\prime}\right)$. Напомним, что $\psi\left(S_{i}\right)=\varphi\left(S_{i}\right)$ для любого положительного термина $S_{i}$ и $\psi\left(P_{j}^{\prime}\right)=\mathbf{D} \backslash \varphi\left(P_{j}\right)$ для любого отрицательного термина $P_{j}^{\prime}$. $\Omega_{1}$-переводом формулы $\Upsilon X_{1} X_{2} \ldots X_{k}$ является силлогистическая формула $\neg S_{1} S_{2} \ldots S_{n} @ P_{1} P_{2} \ldots P_{m}$. Она $\mathcal{W}$-значима, е.т.е. неверно, что $\mathcal{W}$-значима формула $S_{1} S_{2} \ldots S_{n} @ P_{1} P_{2} \ldots P_{m}$, е.т.е. неверно, что $\varphi\left(S_{1}\right) \cap \varphi\left(S_{2}\right) \cap \ldots \cap \varphi\left(S_{n}\right) \cap\left(\mathbf{D} \backslash \varphi\left(P_{1}\right)\right) \cap\left(\mathbf{D} \backslash \varphi\left(P_{2}\right)\right) \cap \ldots \cap\left(\mathbf{D} \backslash \varphi\left(P_{m}\right)\right)=$ $\varnothing$, е.т.е. $\varphi\left(S_{1}\right) \cap \varphi\left(S_{2}\right) \cap \ldots \cap \varphi\left(S_{n}\right) \cap\left(\mathbf{D} \backslash \varphi\left(P_{1}\right)\right) \cap\left(\mathbf{D} \backslash \varphi\left(P_{2}\right)\right) \cap \ldots \cap(\mathbf{D} \backslash$ $\left.\varphi\left(P_{m}\right)\right) \neq \varnothing$. Очевидно, что условия $\mathcal{V}$-значимости $\Upsilon X_{1} X_{2} \ldots X_{k}$ и условия $\mathcal{W}$-значимости ее $\Omega_{1}$-перевода в произвольной модели $\langle\mathbf{D}, \varphi\rangle$ эквивалентны (в силу коммутативности операции $\cap$ ).

В случае, когда $A$ - сложная формула, справедливость теоремы основывается на одинаковой интерпретации пропозициональных связок в обеих логиках, а также на определении перевода $\Omega_{1}$. 
Теперь несложно доказать следующую метатеорему:

Теорема 8. Для любой формулы $A$ языка $\mathbf{C \Upsilon}$ верно, что $A \mathcal{V}$-общезначима, е.т.е. ее перевод $\Omega_{1}(A)$ является $\mathcal{W}$-общезначимым.

Доказателъство. Из формулировки Теоремы 7 по законам логики предикатов вытекает: для любой формулы $A$ языка $\mathbf{C} \boldsymbol{\Upsilon}$ верно, что $\mathcal{V}(A, \mathbf{D}, \varphi)$ в каждой модели $\langle\mathbf{D}, \varphi\rangle$, е.т.е. $\mathcal{W}\left(\Omega_{1}(A), \mathbf{D}, \varphi\right)$ в каждой модели $\langle\mathbf{D}, \varphi\rangle$. Далее просто используем определения $\mathcal{V}$-общезначимой и $\mathcal{W}$-общезначимой формул.

В Маркин, 2004] был обоснован тезис о семантической непротиворечивости и полноте силлогистического исчисления $\mathbf{C \Phi} @$ :

Утверждение 1. Для любой формулы $B$ языка $\mathbf{C \Phi} @$ верно, что $B$ доказуема в $\mathbf{C \Phi} @$, е.т.е. $B \mathcal{W}$-общезначима.

Поскольку $\Omega_{1}$-переводы всех формул языка $\mathbf{C} \boldsymbol{\Upsilon}$ являются формулами языка $\mathbf{C \Phi} @$, в качестве непосредственного следствия из Утверждения 1 получаем:

Утверждение 2. Для любой формулы $A$ языка $\mathbf{C} \mathbf{\Upsilon}$ верно, что формула $\Omega_{1}(A)$ доказуема в $\mathbf{C \Phi} @$, е.т.е. $\Omega_{1}(A) \mathcal{W}$-общезначима.

Докажем, наконец, метатеорему о семантической непротиворечивости и полноте исчисления суждений существования $\mathbf{C} \mathbf{\Upsilon}$ :

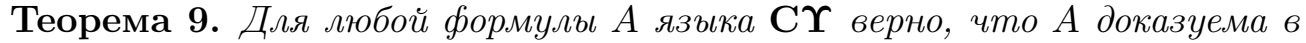
Cr, е.т.е. А является $\mathcal{V}$-общезначимой.

Доказательство. Рассмотрим произвольную формулу $A$ языка $\mathbf{C \Upsilon}$.

Формула $A$ доказуема в $\mathbf{C} \boldsymbol{\Upsilon}$, е.т.е. (по Теореме 5 р) формула $\Omega_{1}(A)$ доказуема в $\mathbf{C \Phi} @$, е.т.е. (согласно Утверждению 2 ) формула $\Omega_{1}(A) \mathcal{W}$-общезначима, е.т.е. (по Теореме 8) формула $A \mathcal{V}$-общезначима. Таким образом,

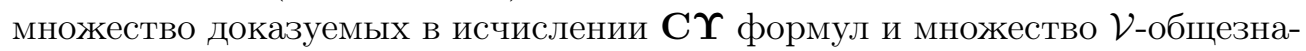
чимых формул равны.

\section{6. Погружение C؟ в исчисление предикатов}

В [Маркин, 2004] был сформулирован перевод ${ }^{\oplus}$ из множества формул языка силлогистики $\mathbf{C \Phi} @$ в язык классического одноместного исчисления предикатов:

$$
\begin{gathered}
\left(S_{1} S_{2} \ldots S_{n} @ P_{1} P_{2} \ldots P_{m}\right)^{\oplus}=\neg \exists x\left(S_{1} x \wedge S_{2} x \wedge \ldots \wedge S_{n} x \wedge \neg P_{1} x \wedge \neg P_{2} x \wedge \ldots \wedge \neg P_{m} x\right), \\
(\neg C)^{\oplus}=\neg C^{\oplus}, \quad(C \nabla D)^{\oplus}=C C^{\otimes} \nabla D^{\oplus} .
\end{gathered}
$$


Была доказана следующая метатеорема:

Утверждение 3. Перевод ${ }^{\oplus}$ погружает $\mathbf{C \Phi} @$ в исчисление предикатов.

Зададим теперь перевод ${ }^{\otimes}$ в первопорядковый язык для формул языка логики суждений существования.

Пусть $u$ - произвольная конечная последовательность общих терминов. Пусть $S_{1}, S_{2}, \ldots, S_{n}$ - результат вычеркивания из $u$ всех отрицательных терминов, а $P_{1}^{\prime}, P_{2}^{\prime}, \ldots, P_{m}^{\prime}$ - результат вычеркивания из $u$ всех положительных терминов. Тогда

$$
\begin{gathered}
(\Upsilon u)^{\otimes}=\exists x\left(S_{1} x \wedge S_{2} x \wedge \ldots \wedge S_{n} x \wedge \neg P_{1} x \wedge \neg P_{2} x \wedge \ldots \wedge \neg P_{m} x\right), \\
(\neg C)^{\otimes}=\neg C^{\otimes}, \quad(C \nabla D)^{\otimes}=C^{\otimes} \nabla D^{\otimes} .
\end{gathered}
$$

Покажем, что доказуемость формулы в системе $\mathbf{C} \boldsymbol{\Upsilon}$ равносильна доказуемости ее ${ }^{\otimes}$-перевода в исчислении предикатов:

Теорема 10. Перевод ${ }^{\otimes}$ погружает $\mathbf{C} \boldsymbol{\Upsilon}$ в исчисление предикатов.

Доказателъство. Поскольку $\Omega_{1}$ погружает исчисление $\mathbf{C} \boldsymbol{\Upsilon}$ в $\mathbf{C \Phi} @$ (Теорема 5), а $\oplus^{\oplus}$ погружает $\mathbf{C \Phi} @$ в исчисление предикатов (Утверждение 3), постольку $\mathbf{C} \mathbf{\Upsilon}$ погружается в исчисление предикатов посредством композиции переводов $\Omega_{1}$ и ${ }^{\oplus}$. Остается доказать, что композиция $\Omega_{1}$ и $^{\oplus}$ равносильна переводу ${ }^{\otimes}$.

Докажем, иными словами, что для любой формулы $A$ языка $\mathbf{C} \mathbf{\Upsilon}$ верно, что формула $A^{\otimes} \equiv \Omega_{1}(A)^{\oplus}$ доказуема в исчислении предикатов. Используем возвратную индукцию по числу пропозициональных связок в $A$.

Пусть $A$ - атомарная формула языка $\mathbf{C}$. Тогда она имеет вид $\Upsilon u$ (точное описание $u$ дано при задании переводов $\Omega_{1}$ и $\left.{ }^{\otimes}\right) . \Omega_{1}(\Upsilon u)^{\oplus}=$ $\left(\neg S_{1} S_{2} \ldots S_{n} @ P_{1} P_{2} \ldots P_{m}\right)^{\oplus}=\neg\left(S_{1} S_{2} \ldots S_{n} @ P_{1} P_{2} \ldots P_{m}\right)^{\oplus}=\neg \neg \exists x\left(S_{1} x \wedge S_{2} x \wedge\right.$ $\left.\ldots \wedge S_{n} x \wedge \neg P_{1} x \wedge \neg P_{2} x \wedge \ldots \wedge \neg P_{m} x\right)$. Последняя формула есть не что иное, как $\neg \neg \Upsilon u^{\otimes}$. Очевидно, что формула $\Upsilon u^{\otimes} \equiv \neg \neg \Upsilon u^{\otimes}$ доказуема в исчислении предикатов.

Пусть $A-$ сложная формула. Ограничимся случаями, когда $A$ имеет вид $\neg C$ и $C \wedge D$. По индуктивному допущению формулы $C^{\otimes} \equiv \Omega_{1}(C)^{\oplus}$ и $D^{\otimes} \equiv \Omega_{1}(D)^{\oplus}$ доказуемы в исчислении предикатов. Но тогда в нем также доказуемы формулы $\neg C^{\otimes} \equiv \neg \Omega_{1}(C)^{\oplus}$ и $\left(C^{\otimes} \wedge D^{\otimes}\right) \equiv \Omega_{1}(C)^{\oplus} \wedge \Omega_{1}(D)^{\oplus}$. Используя определения трех переводов в этих записях, получаем: формулы $(\neg C)^{\otimes} \equiv \Omega_{1}(\neg C)^{\oplus}$ и $(C \wedge D)^{\otimes} \equiv \Omega_{1}(C \wedge D)^{\oplus}$ доказуемы в исчислении предикатов. 


\section{7. Заключение}

Предложенная в этой статье логика суждений существования может быть использована не только для сопоставления с достаточно экзотической силлогистикой с неопределенно-местной константой, но и для исследования обычных силлогистических теорий, язык которых содержит стандартные бинарные константы (напр., $a, e, i, o)$. K настоящему времени построены многие системы позитивной и негативной силлогистик. С семантической точки зрения они различаются условиями истинности и ложности форм атрибутивных суждений, причем сами эти условия обычно формулируются (или могут быть эквивалентным образом переформулированы) в терминах суждений существования. Поэтому возникает возможность различным образом определять бинарные силлогистические константы посредством константы $\Upsilon$. При этом мы получаем разные дефинициалъные расширения логики C؟, а каждая конкретная силлогистика может рассматриваться как своего рода фрагмент логики суждений существования.

Сформулируем описанную идею в более точных терминах. Пусть Df система определений силлогистических констант в языке логики $\mathbf{C} \Upsilon$. Под $\mathbf{C} \boldsymbol{\Upsilon}+\mathbf{D f}$ будем понимать дефинициальное расширение $\mathbf{C} \boldsymbol{\Upsilon}$ посредством системы определений $\mathbf{D f}$. Исчисление $\mathbf{S}$, сформулированное в языке силлогистики, назовем силлогистическим фрагментом C؟ при определениях $\mathbf{D f}$, е.т.е. для любой формулы $A$ языка $\mathbf{S}$ верно: $A$ доказуема в $\mathbf{S}$, е.т.е. $A$ доказуема в $\mathbf{C} \boldsymbol{\Upsilon}+\mathbf{D f}$.

Приведем два примера, касающихся вступительной части этой статьи. Силлогистическим фрагментом $\mathbf{C} \Upsilon$ при брентановских определениях $a$, $e, i$, o является известная система фундаментальной силлогистики СФ. А силлогистическим фрагментом $\mathbf{C} \Upsilon$ при кэрролловских определениях $a$, $e, i$ будет некоторая формализация силлогистики Кэрролла.

Еще одной важной задачей представляется построение аналитикотабличного варианта логики суждений существования. Такого рода представление этой логической теории может дать эффективный метод установления законов и форм правильных рассуждений в силлогистиках с альтернативными трактовками атрибутивных суждений.

\section{Литература}

Маркин, 2004 - Маркин В.И. Фундаментальная силлогистика с неопределенноместной константой // Логические исследования. Вып. 11. М.: Наука, 2004. C. 187-199.

Маркин, 2021 - Маркин В.И. Логика суждений существования, рекурсивно эквивалентная силлогистике с неопределенно-местной константой // Двенадцатые Смирновские чтения: материалы Международной научной конференции, 
Москва (24-26 июня 2021 г.). М.: Русское общество истории и философии науки, 2021. С. 112-117.

Смирнов, 2002 - Смирнов В.А. Логические методы анализа научного знания. М.: Эдиториал УРСС, 2002. $263 \mathrm{c}$.

Brandl, 2018 - Brandl J.L. Brentano's Theory of Judgement // The Stanford Encyclopedia of Philosophy. URL = https://plato.stanford.edu/entries/brentanojudgement/

Carroll, 1896 - Carroll L. Symbolic Logic. London: Macmillan and Co., 1896. 200 p. 


\title{
VLADIMIR I. MARKIN
}

\section{Logic of existence judgements and syllogistic}

\author{
Vladimir I. Markin \\ Lomonosov Moscow State University, \\ 27/4 Lomonosovskiy prospect, Moscow, 119991, Russian Federation. \\ E-mail: markin@philos.msu.ru
}

\begin{abstract}
We set out a formal system for logical analyses of existence judgements. Its language contains the constant of existence, atomic formulas are formed by the concatenation of this constant with any finite sequence of general terms (positive and negative). Complex formulas are formed by means of propositional connectives. We formulate a natural semantics for this language. The extension of a positive general term in a model is a subset of the domain, the extension of a negative general term is the complement to the extension of the respective positive term. An atomic formula is valid in a model iff the intersection of the extensions of all terms in it is non-empty. We set out an axiomatization of the set of generally valid formulas. This calculus formulates on the base of classical propositional logic. We notice that categorical judgements can be defined with the aid of existence judgements and raise a question about metatheoretic interrelations among our logic and different syllogistic theories. We prove that the logic of existence judgements is recursively equivalent to the syllogistic with indefinably-placed constant which is the generalization of constant $a$. We formulate the embedding translations from each of these two systems to another. Also, we prove soundness and completeness theorems for the calculus of existence judgements.
\end{abstract}

Keywords: existence judgements, categorical judgements, syllogistic, logical calculus, semantics, embedding translation

For citation: Markin V.I. "Logika suzhdenii sushchestvovaniya i sillogistika" [Logic of existence judgements and syllogistic], Logicheskie Issledovaniya / Logical Investigations, 2021, Vol. 27, No. 2, pp. 31-47. DOI: 10.21146/2074-1472-2021-27-2-31-47 (In Russian)

\section{References}

Brandl, 2018 - Brandl, J.L. Brentano's Theory of Judgement, in: The Stanford Encyclopedia of Philosophy. URL = https://plato.stanford.edu/entries/brentanojudgement/.

Carroll, 1896 - Carroll, L. Symbolic Logic. London: Macmillan and Co., 1896. 200 pp.

Markin, 2004 - Markin V.I. "Fundamental'naya sillogistika s neopredelenno-mestnoi konstantoi" [Fundamental syllogistic with indefinably-placed constant], in: Logicheskie issledovaniya [Logical Investigations]. Vol. 11. Moscow: Nauka, 2004, pp. 187-199. (In Russian)

Markin, 2021 - Markin, V.I. "Logika suzhdenii sushchestvovaniya, rekursivno ekvivalentnaya sillogistike s neopredelenno-mestnoi konstantoi" [Logic of existence 
judgements that is recursively equivalent to syllogistic with indefinably-placed constant], in: Dvenadtsatye Smirnovskie chteniya: materialy Mezhdunarodnoi nauchnoi konferentsii, Moskva (24-26 iyunya 2021 g.) [Twelfth Smirnovs Readings: Proceedings of the international scientific conference, Moscow (June 24-26, 2021)]. Moscow: Russkoe obshchestvo istorii i filosofii nauki, 2021, pp. 112-117. (In Russian)

Smirnov, 2002 - Smirnov, V.A. Logicheskie metody analiza nauchnogo znaniya [Logical methods of the analyses of scientific knowledge]. Moscow: Editorial URSS, 2002. 263 pp. (In Russian) 